\title{
Possível influência de raios cósmicos galácticos sobre a variação de temperatura na latitude de $\sim 30$ 오 no estado do Rio Grande do Sul no último século
}

\author{
Pedro Cruz de Moura Lima*, Everton Frigo, Moises Razeira, Universidade Federal do Pampa
}

Copyright 2014, SBGf - Sociedade Brasileira de Geofísica

Este texto foi preparado para a apresentação no VI Simpósio Brasileiro de Geofísica, Porto Alegre 14 a 16 de outubro de 2014. Seu conteúdo foi revisado pelo Comitê Técnico do VI SimBGf, mas não necessariamente representa a opinião da SBGf ou de seus associados. É proibida a reprodução total ou parcial deste material para propósitos comerciais sem prévia autorização da SBGf.

\begin{abstract}
In this work we investigate the possible connection between galactic cosmic rays (GCR) and annual mean temperature variations in south Brazil through spectral analysis. Temperature data used were measured in three meteorological stations located next to the latitude of $30^{\circ}$ $S$ in the cities of Porto Alegre (POA), Santa Maria (SMA) and Uruguaiana (URU), in a region heavily affected by the South Atlantic Magnetic Anomaly (SAMA). Results shown the occurrence of almost simultaneous events of maximum temperature peaks separated for $\sim 20$ years. Spectral analysis revealed periodicities of $\sim 11$ and $\sim 22$ years, characteristic of GCR, in temperature data. Furthermore, spectral coherence analysis results indicated that GCR and climate are not related by persistent linear mechanisms.
\end{abstract}

\section{Introdução}

Nas ultimas décadas um assunto que vem ganhando espaço nas discussões científicas devido a sua influência na sociedade e seu desenvolvimento são as mudanças climáticas. Sabemos devido a dados históricos e registros geológicos que o clima da terra esta em constante mudança, que por alguns pesquisadores são atribuídas à ação antrópica, e por outros, como resultado de fenômenos físicos naturais originados interna e externamente ao nosso planeta, como erupções vulcânicas ou mudanças na configuração orbital. Em meio a estas duas linhas de pensamento vemos que as origens destas mudanças ainda não são bem compreendidas e requerem ainda muita investigação devido a sua complexidade e grande número de variáveis e condicionantes.

Uma das linhas de pesquisa sobre as origens das variações climáticas estudam a atividade solar e sua interação com a terra como genealogia destes eventos, pois qualquer alteração na radiação recebida do Sol ou perdida para o espaço tende a afetar o clima. Ainda assim, os estudos relacionados à atividade solar mostram um comportamento cíclico de variação de atividade solar de aproximadamente 11 anos e um secundário atribuído à inversão de polaridade do campo magnético de cerca de 22 anos, escalas de tempo que por muitos autores é considerada insuficiente devido a quantidade de energia envolvida para causar alterações significativas.
Entretanto, alguns autores sugerem um mecanismo que relaciona indiretamente a atividade solar com as mudanças climáticas através do fluxo de GCR, segundo Dickinson (1975), Svensmark \& Friis-Christensen (1997) e Svensmark (2007). Os GCR são modulados pelos campos magnéticos do sol e da terra que em determinados períodos e ou regiões favorecem a entrada destas partículas de alta energia em nossa atmosfera, podendo influenciar na cobertura de nuvens baixas, que tem um papel muito importante no sistema climático terrestre. Períodos com grande fluxo de GCR estariam associados à maior cobertura de nuvens baixas e consequentemente diminuição da temperatura da superfície terrestre e quando diminuído o fluxo de GCR ocorre o efeito contrário. Porém existem outros condicionantes que fazem com que tenhamos efeitos distintos em diferentes localidades e épocas. Ao trabalharmos com escalas de tempo decadal a centenal, temos que os GCR têm a sua variabilidade condicionada pela intensidade do campo magnético solar, que tem duas principais componentes cíclicas de 11 e 22 anos e chegando ao nosso planeta tem como condicionante a intensidade e direção do campo magnético terrestre, tendo uma maior entrada nas regiões polares onde o campo é predominantemente vertical e menor na região equatorial onde o CMT é predominantemente horizontal (Frigo, 2013). Em relação à intensidade, regiões com baixa intensidade favorecem a entrada dos GCR, como é o caso da região influenciada pela SAMA.

Neste trabalho, usando dados de temperatura média anual, (calculados através da média aritmética entre as médias das temperaturas máximas e mínimas anuais) entre 1932 e 2009 de estações meteorológicas situadas no Rio Grande do Sul, localizadas numa linha de latitude de $\sim 30^{\circ} \mathrm{S}$, na região fortemente afetada pela SAMA nos últimos cem anos, foram investigados os possíveis efeitos climáticos coligados ao fluxo de GCR nesta região peculiar.

\section{Problema Investigado e Metodologia}

A área de estudo compreende a região onde estão situadas as estações meteorológicas das cidades de Porto Alegre, Santa Maria e Uruguaiana, todas próximas a trajetória do centro da SAMA e fortemente influenciadas por este fenômeno desde o inicio do século XX. A SAMA ocupa atualmente parte do oceano Atlântico Sul e do continente Sul-Americano, com valores de intensidade total do campo geomagnético inferiores a $25.000 \mathrm{nT}$. Os valores de menor intensidade da SAMA, determinada como o seu centro, têm diminuído ao decorrer dos anos e migrado a sua posição com direção predominante para oeste. Estudos publicados por autores como König et al. 
(1978) e Smart \& Shea (2009) mostram que os valores de rigidez magnética de corte vertical, que indica a energia mínima que uma partícula eletricamente carregada deve possuir para penetrar a barreira imposta pelo campo geomagnético, vem diminuindo ao longo do ultimo século como efeito das variações geomagnéticas associadas a SAMA.

A modulação climática pela atividade solar dentro de escalas decadais e multidecadais foi proposta por alguns autores e vem sendo investigada com diferentes metodologias. Usando dados de médias anuais de temperatura no período de 1932 a 2009 das estações meteorológicas que são apresentadas na Figura 1 e os dados das forçantes climáticas propostas apresentados na Figura 2, foi investigado a presença de sinais associados à variabilidade dos GCR.

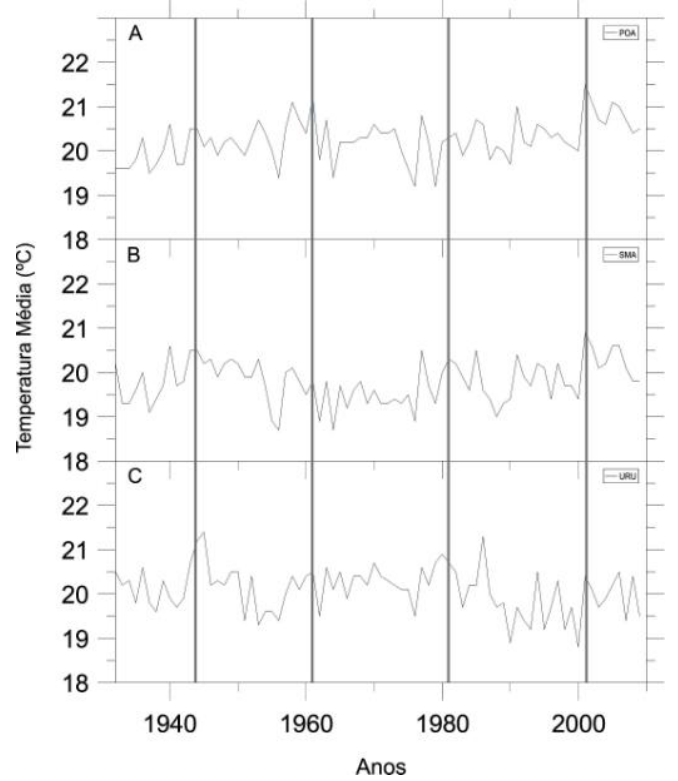

Figura 1 - Séries temporais das temperaturas médias anuais das estações meteorológicas: POA (A), SMA (B), $U R U(C)$. As bandas sombreadas indicam eventos de máxima temperatura ocorridos simultaneamente.

Os dados sugeridos como forçantes climáticas relacionados com o fluxo de GCR são a série temporal do número de manchas solares (Fig. 2A), apresentada em termos de Rz (Hoyt e Schatten, 1997), que foi obtido no site (http://sidc.oma.be/). O ciclo de Hale (Fig. 2C) tratase do número de manchas solares multiplicado por -1 em ciclos ímpares, representado como Rz22. Já a série temporal de contagem de nêutrons (Fig.2B), indicador de GCR, esta disponível para o período de 1953 a 2006 no site da Universidade de New Hampshire, onde neste conjunto de dados, a transição dos ciclos solares pode ser notada com a forma triangular mais fina para a transição dos ciclos solares 19 para 20 e 21 para 22, e, um formato mais espesso e quadrado para as transições dos ciclos solares 20 para 21 e 22 para 23 anos. Os dados de GCR são afetados pelo campo magnético solar tendo uma modulação de 11 anos relacionado à intensidade do campo e de 22 relacionado à inversão do campo magnético solar.

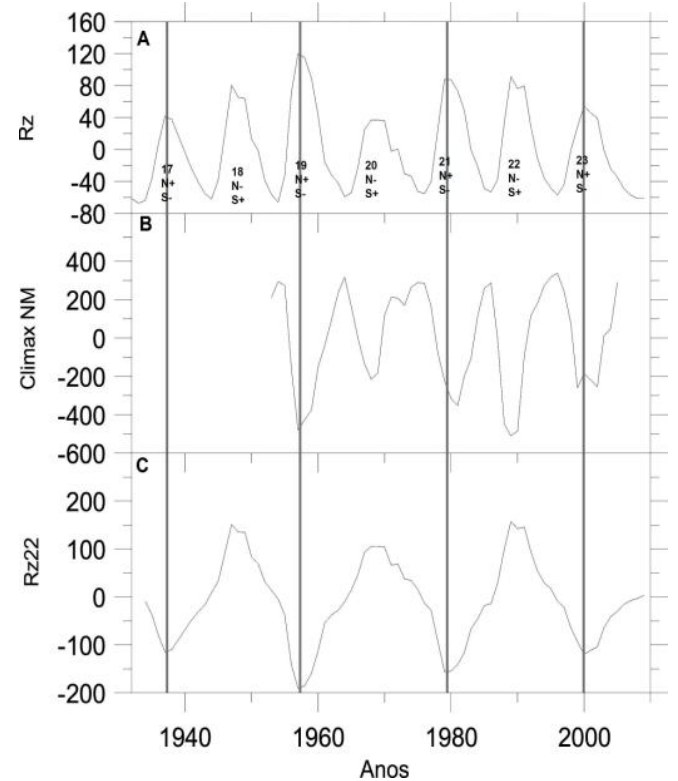

Figura 2 - Série temporal $R z(A)$, contagem de nêutrons no Observatório Climax (B) e série temporal Rz22 (C). As bandas sombreadas indicam os máximos dos ciclos solares impares.

Em busca das periodicidades de interesse dentro dos dados de temperatura, foi utilizado o método clássico ARIST (Análise por Regressão Iterativa de Séries Temporais). Este método permite determinar as periodicidades presentes nos dados fornecendo também a significância estatística dos resultados. O ARIST é um método iterativo que busca três parâmetros desconhecidos (frequência, amplitude, fase), visando minimizar o quadrado da chamada função condicional, que é a diferença entre os dados medidos e um ajuste de uma função senoidal a estes dados medidos.

Para estimar a coerência espectral entre os dados de temperatura e forçantes climáticas foi utilizada a análise de WTC, técnica que permite identificar as periodicidades de maior covariância comum entre as duas séries temporais considerando ambos os espectros normalizados. A WTC utiliza como base a ondaleta de Mortet e os resultados fornecem valores de coerência entre 0 e 1, permitindo analizar a coerência em tempo e frequência (Torrence e Compto,1998). Neste trabalho foi utilizado o código em MATLAB proposto por Grinsted et al. (2004), que permite estimar também os ângulos de fase entre as duas séries temporais analisadas. Ângulos de 0 e 180 graus indicam uma relação linear entre as séries, estando em fase ou em anti-fase. Outras configurações indicam relações não lineares.

\section{Resultados}

Observamos na Figura 1, onde são apresentadas as séries temporais das médias de temperatura das 
estações meteorológicas de POA, SMA e URU, um comportamento muito similar entre as séries temporais, esperado devido à proximidade entre as estações e por estarem em uma mesma latitude, sendo diferenciadas apenas por aspectos regionais e pela continentalidade e maritimidade. Na Figura 1, assim como na Figura 2, bandas sombreadas foram colocadas para facilitar a visualização da possível relação entre os eventos de valores máximos para as médias anuais de temperatura, com os mínimos de Rz22, conforme sugerido para algumas estações meteorológicas por Frigo et al. (2013).

Os valores máximos encontrados nos dados de temperatura foram 1940-1945, 1959-1961, 1977-1980 e 2001 , intervalos de tempo muito próximos dos mínimos de Rz22 e ocorrendo muito próximo dos picos de máximo dos ciclos solares ímpares dos anos de 1937, 1957, 1979 e 2000, sugerindo a modulação climática pelos GCR/atividade solar para periodicidades de $\sim 22$ anos.

Com os dados de temperatura e das forçantes climáticas livre de tendências, o método de regressão interativa ARIST foi aplicado com o intuito de procurar as periodicidades presentes nos dados. Os resultados, para periodicidades de $\sim 11$ e de $\sim 22$ anos, são apresentados na Tabela 1 com um nível de confiança de 95\%.

Tabela 1 - Períodos estatisticamente significativos encontrados nos dados utilizados.

\begin{tabular}{ccc}
\hline \multicolumn{3}{c}{ Período (amplitude do sinal) } \\
\hline Dados & $8-15$ anos & $19-25$ anos \\
\hline POA & $10.97(0.172)$ & - \\
SMA & $10.61(0.160), 12.18(0.161)$ & $19.02(0.198)$ \\
URU & $8.66(0.171), 12.18(0.183)$ & $19.02(0.277)$ \\
Rz & $8.70(21.183), 10.59(63.862)$ & - \\
Rz22 & $14.59(32.043)$ & $21.21(115.329)$ \\
Nêutrons $8.10(123.212), 10.56(298.037)$ & $23.62(127.928)$ \\
\hline
\end{tabular}

Todas as estações meteorológicas apresentaram periodicidades entre 2 e 7 anos que possivelmente estejam relacionadas ao fenômeno El Niño Oscilação Sul (El Niño Southern Oscilattion - ENSO), que não está sendo investigado neste trabalho. A periodicidade entre 8 e 15 anos característica do ciclo de Schwabe esteve presente em todos os conjuntos de dados analisados, já a periodicidade referente ao ciclo de Hale (19 a 25 anos), não aparece nos dados de temperatura de POA. Os dados das estações de SMA, URU apresentam periodicidade de $\sim 33$ anos, possivelmente ligado ao ciclo solar de Brückner.

Para verificar a coerência espectral entre os dados de temperatura e as séries de $R z$ e $R z 22$ foi realizada a análise WTC. A Figura 3 apresenta o espectro de WTC entre temperatura e $\mathrm{Rz}$, referente ao ciclo de 11 anos, sinal este que se mostrou estatisticamente coerente após 1950 para POA e SMA com sinal mais forte próximo ao ano de 1990 com valores de coerência acima de 0,8 . Já para URU o sinal característico do ciclo de Schwabe só aparece em meados dos anos 2000 e com valores de coerência de no máximo 0,7 . O ângulo de fase indica uma relação não linear, porém com comportamento similar entre POA e SMA principalmente depois de 1990.

A coerência entre os dados de temperatura e a série de Rz22 é apresentada na Figura 4, onde a periodicidade de 22 anos, característica do ciclo de Hale, aparece de modo distinto para as três estações meteorológicas. Para os dados de POA o sinal aparece durante toda a série com os valores mais altos de coerência $(\sim 0,7)$ para as décadas de 50, 60 e 70. Em SMA o sinal aparece de forma intermitente, com intervalo de omissão entre 1960 e 1985. Assim como em POA, os dados referentes à URU apresentam sinal contínuo de 22 anos durante toda a série temporal, porém com altos valores de coerência para toda a série (valores superiores a 0,8 após 1990). Mesmo apresentando-se de modo desigual para os conjuntos de dados, as setas que indicam os ângulos de fase aparecem de modo similar com a tendência de apontar para cima até 1980 quando assumem a tendência de apontar para a esquerda, em alguns pontos indicando uma relação de anti-fase. Periodicidades de até quatro anos aparecem em todas as estações de forma intermitente, com maior intensidade em POA e SMA a partir dos anos 70 até aproximadamente final dos anos 90 , com alta coerência e configuração similar dos ângulos de fase indicando que os sinais estão parte em fase e parte próximo disso. Periodicidades de $\sim 7$ anos aparecem entre 1950 a 1960 em POA e SMA sem indicativos de linearidade e, aparece até 1990 para URU com maior coerência para o período de 1970 a 1990, indicando relação linear de anti-fase entre as séries temporais para esta faixa de periodicidades.

\section{Discussão e Conclusão}

Periodicidades possivelmente associadas ao fenômeno ENSO estão presentes em todas as séries temporais de temperatura analisadas. O sinal característico do ciclo solar de $\sim 11$ anos apresenta comportamento similar para POA e SMA tanto em questão de fase, como em valores de coerência, de $\sim 0,8$ a partir da década de 90 , porém com parte do sinal fora do COI. Para a estação de URU o sinal de $\sim 11$ anos aparece apenas após o ano de 2000, com altos valores de coerência fora do $\mathrm{COI}$ e assumindo valores pouco expressivos, ou seja, comportamento bem diferente das outras duas estações meteorológicas.

Assim, notamos através das análises de coerência, que as estações de POA e SMA têm um comportamento muito similar para os sinais associados ao ciclo de Schwabe, ao contrário da estação de URU. Esse fato indica um comportamento regional que não é acompanhado pela estação de URU, localizada na parte mais distal do continente, possível causa deste comportamento diferenciado. Esta conclusão vai de encontro a trabalhos anteriores que sugerem que os sinais da atividade solar / GCR e ENSO podem aparecer com diferentes características em locais distintos e sob distintas variáveis meteorológicas (Grimm et al., 2000; Barros et al., 2002; Haigh, 2007; Gray et al., 2010). 
O ciclo de 22 anos, associado à variabilidade dos GCR, nas figuras 1 e 2, aparece como um possível modulador climático indicado pela proximidade entre os eventos de valores mínimos de Rz22, máximos das médias de temperatura anual e mínimos das contagens de nêutrons. Os valores de mínimos de Rz22 ocorreram em 1937, 1957, 1979 e 2000. Os máximos de temperatura ocorreram em 1940, 1945 (URU), 1959 (SMA), 1961, 1977, 1986 (URU) e 2001. Os valores mínimos de contagens de nêutrons ocorreram em 1958, 1982 e 2001 -2002 .
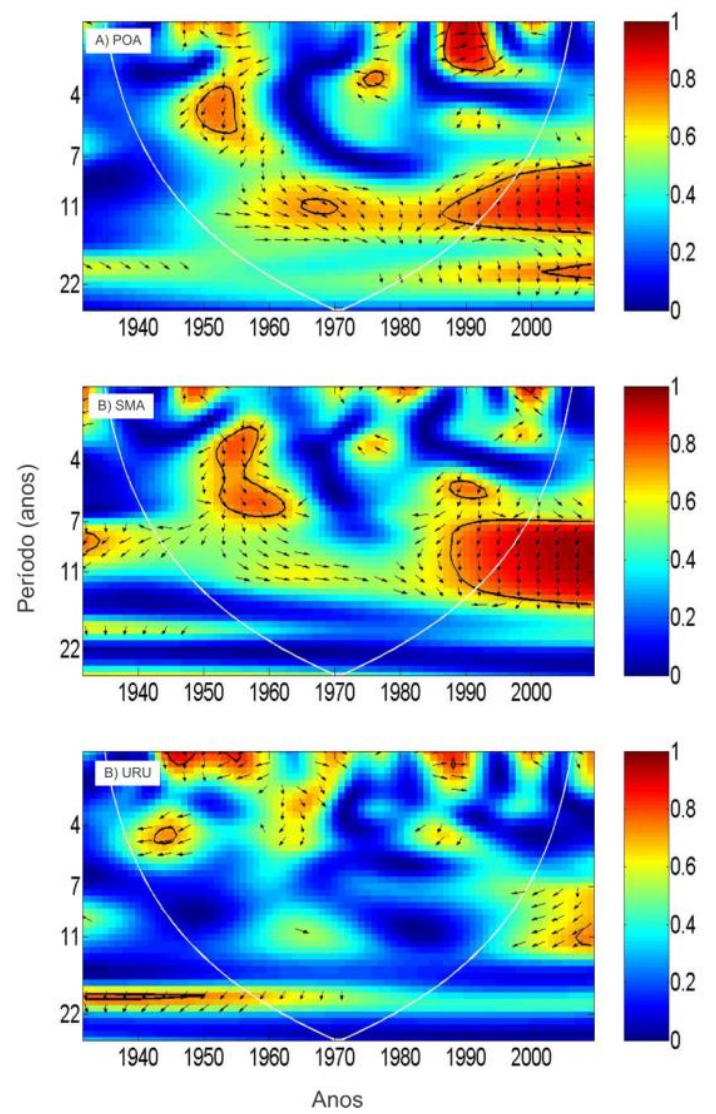

Figura 3 - Espectro de coerência wavelet entre a série temporal $R z$ e as séries de temperaturas das estações POA (A), SMA (B) e URU (C). Os valores de coerência estão indicados pelas cores. A curva branca em formato de $U$ indica o cone de influência (COI), abaixo da qual efeitos de borda não podem ser desprezados. A linha preta indica o limite de confiança de $95 \%$ para os valores de coerência. As setas pretas indicam os ângulos de fase.

O sinal de 22 anos aparece para dados de URU e SMA, porém na análise ARIST esta periodicidade não aparece com significância estatística para POA. Além disso, nas análises de coerência WTC a correlação se mostrou fraca para POA e intermitente para SMA com o sinal fora do COI.

O sinal contínuo em duas das três estações e os valores de máxima temperatura média ocorrendo em datas próximas a eventos de mínimos de Rz22 vão de encontro a trabalhos de outros autores como Rampelotto et al (2012) e Frigo et al. (2013), que indicam os GCR como possíveis gatilhos de mecanismos na criação de nuvens baixas e consequentemente nos valores de temperatura na região do SAMA. Em contra partida mais estudos em escala regional, em outras localidades se fazem necessários principalmente para melhor compreensão do comportamento apresentado por SMA para o sinal do ciclo de Hale, por estar localizada na mesma latitude das demais estações, em área afetada pela SAMA e entre estações que apresentaram comportamento próximo.
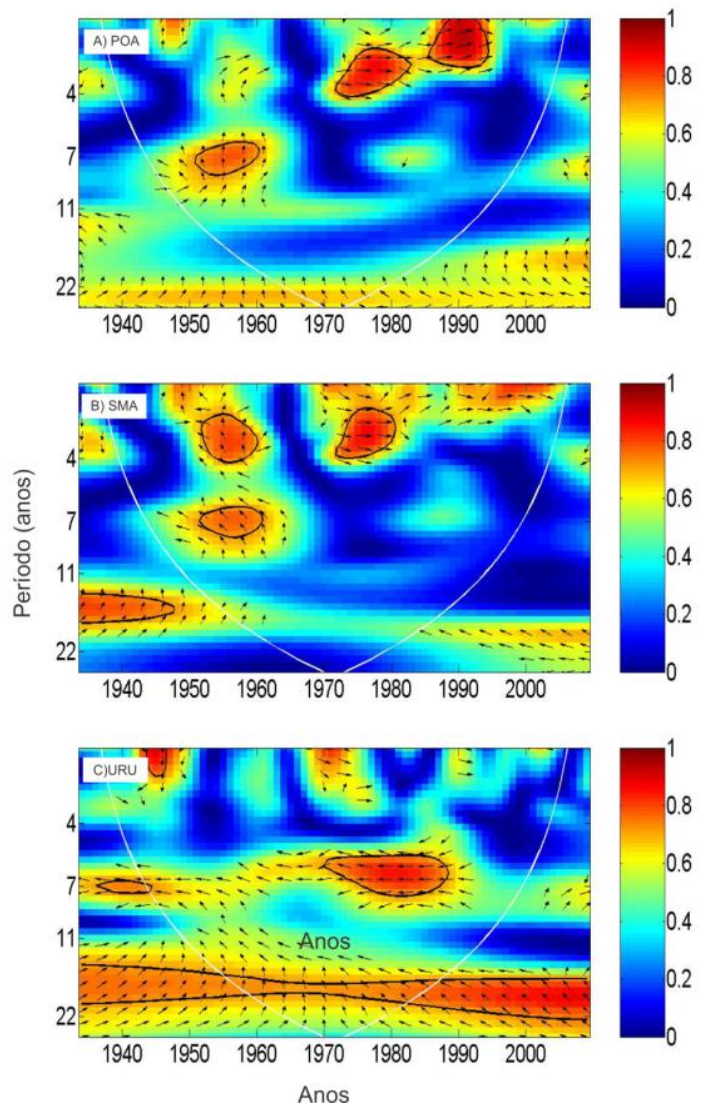

Figura 4 - Espectro de coerência wavelet entre a série temporal Rz22 e as séries de temperaturas das estações POA (A), SMA (B) e URU (C). A legenda é similar a da Figura 3.

\section{Agradecimentos}

Os autores agradecem ao Instituto Nacional de Meteorologia (INMET), por fornecer os dados de temperatura das estações meteorológicas de Porto Alegre, Santa Maria e Uruguaiana e também a National Science Foundation (Grant ATM-0339527) por disponibilizar os dados de contagem de nêutrons. Pedro Cruz de Moura Lima agradece à Universidade Federal do Pampa pela concessão da Bolsa PBDA. 


\section{Referências}

Barros, V. R., Grimm, A. M., and Doyle, M. E.: Relationship between temperature and circulation in Southeastern South America and its influence from El Niño and La Niña events, J. Meteorol.Soc. Jpn., 80-1, 3344, 2002.

Dickinson, R.E., 1975. Solar variability and the lower atmosphere. Bull. Amer. Meteorol. Soc., 56, 1240-1248.

Frigo,et al ., 2013. Evidence for cosmic ray modulation in temperature records fromthe South Atlantic Magnetic Anomaly region. Ann. Geophys., 31, 1833-1841, 2013.

Grinsted, A., Moore, J. C., and Jevrejeva, S.: Application of the cross wavelet transform and wavelet coherence to geophysical time series, Nonlinear Proc. Geoph., 11, 561-566, 2004.

Gray, L. J., Beer, J., Geller, M., Haigh, J. D., Lockwood, M.,Matthes, K., Cubasch, U., Fleitmann, D., Harrison, G., Hood,L., Luterbacher, J., Meehl, G. A., Shindell, D., Van Geel, B., and White, W.: Solar influence on climate, Rev. Geophys., 48,RG4001, doi:10.1029/2009RG000282, 2010.

Grimm, A., Barros, V., and Doyle, M.: Climate variability in southernSouth America associated with El Niño La Niña events, J. Climate, 13, 35-58, 2000.

Haigh, J. D.: The Sun and The Earth's Climate, Living Reviews in Solar Physics, 4, 2007.

Hoyt, D. V. and Schatten, K. H.: The role of the sun in climate change, Oxford University Press, New York, 1997.

König, P. J., Walt, A. J. V. D., Stoker, P. H., Raubenheimer, B. C.,Shea, M. A., and Smart, D. F.: Vertical cutoff rigidity and the intensity distribution of cosmic rays near Cape Town, in: International Cosmic Ray Conference, 15th, Plovdiv, Bulgaria, 13- 26 August 1977, Conference Papers, Vol. 4, A79-37301 15-93, Sofia, Bulgarian Academy of Sciences, 173-177, 1978.

Rampelotto, P. H., Rigozo, N. R., da Rosa, M. B., Prestes, A.,Frigo, E., Souza Echer, M. P., and Nordemann, D. J. R.: Variabilityof Rainfall and Temperature (1912-2008) from SantaMaria (29_41' S, 53 48' W) and its Connection with Natural Influences,

J. Atmos. Solar-Terr. Phys., 77, 152-160, 2012.

Smart, D. F. and Shea M. A.: Fifty years of progress in geomagnetic cutoff rigidity determinations, Adv. Space Res., 44, 1107-1123,2009.

Svensmark, H., 2007. Cosmoclimatology: a new theory emerges. News Rev. Astron. Geophys., 48, 1.18-1.24.
Svensmark, H., and Friis-Christensen, E., 1997. Variation of Cosmic Ray Flux and Global Cloud Coverage - a Missing Link in Solar-Climate relationships. J. Atmos. Solar-Terr. Phys., 59, 1225-1232.

Torrence, C., Compo, G.P., 1998. A practical guide to wavelet analysis. Bull. Am. Meteorol. Soc., 79, 61-78. 